\title{
INVESTIGACIÓN
}

Recibido: 31/05/2019 --- Aceptado: 19/06/2019 --- Publicado: 15/03/2020

\section{DIAGNÓSTICO DE LA CONSTRUCCIÓN TEÓRICA DE INVESTIGACIONES EN COMUNICACIÓN EN MÉXICO}

\section{Diagnosis of the theoretical construction of Communication research in Mexico}

Irma Mariana Gutiérrez Morales¹. Universidad Nacional Autónoma de México. México.

mariana_gmx@yahoo.com

Luis Antonio Aguilar Ramírez. Universidad Nacional Autónoma de México.

México.

1_aguilaramirez@yahoo.com.mx

Irma Leticia González Chaparro. Universidad Nacional Autónoma de México. México.

igtrabajo@yahoo.com.mx

Angélica Montserrat Pérez Lima. Universidad Nacional Autónoma de México. ampl.monrock@gmail.com

María Guadalupe Sandoval Pantoja. Universidad Nacional Autónoma de México. México.

sandovalmagu@gmail.com

Benito Ilich Suárez Bedolla. Universidad Nacional Autónoma de México. México. benitoilich@gmail.com

\section{RESUMEN}

El propósito de este artículo es conocer el estado actual que guarda la construcción teórica de investigaciones en comunicación en México, mediante la identificación y análisis de objetos de estudio y teorías que se manejan en 90 tesis del Programa de Posgrado en Ciencias Políticas y Sociales de la UNAM, con orientación en Comunicación, elaboradas desde el año 2012 a la fecha y publicadas en el sistema

\footnotetext{
${ }^{1}$ Irma Mariana Gutiérrez Morales: Doctora en Ciencias Políticas y Sociales por la Universidad Nacional Autónoma de México. Profesora de tiempo completo de las Licenciaturas en Comunicación y Pedagogía y de la Maestría en Comunicación de la Facultad de Estudios Superiores Acatlán, UNAM.
} 
Gutiérrez Morales, I. M.; Aguilar Ramírez, L. A.; González Chaparro, I. L.; Pérez

Lima, A. M.; Sandoval Pantoja, M. G. y Suárez Bedolla, B. I.

Diagnóstico de la construcción teórica de investigaciones en Comunicación en México

TESIUNAM. Se trata de un estudio cuantitativo que desarrolla un análisis a partir de 16 reactivos, para establecer las incidencias de líneas de investigación y temas; elementos del diseño de investigación; marcos teóricos, autores y obras de referencia; enfoques metodológicos y técnicas; tipos de resultados y grado de construcción teórica original. Los datos obtenidos enfatizan la dispersión de temas, la preferencia por los enfoques cualitativos en dos de cada tres tesis realizadas y por determinados autores y obras de referencia; así como el bajo nivel de crítica o construcción teórica original, aun en las tesis de nivel doctorado.

PALABRAS CLAVE: marcos teóricos - objetos de estudio - metodología - objetivos - hipótesis - técnicas - investigación en comunicación - UNAM - tesis.

\section{ABSTRACT}

The purpose of this article is to know the current state of theoretical construction of communication research in Mexico, through identification and analysis of objects of study and theories that are handled in 90 theses of the Programa de Posgrado en Ciencias Politicas y Sociales, UNAM, which were elaborated from the year 2012 to the date and were published in TESIUNAM system. This is a quantitative study that develops an analysis based on 16 reagents, to establish the incidences of lines of research and topics; elements of research design; theoretical frameworks, authors and reference works; methodological approaches and techniques; types of results and degree of original theoretical construction. Data obtained emphasize the dispersion of themes, the preference for qualitative approaches and for certain authors and reference works; as well as the low level of criticism or original theoretical construction, even in $\mathrm{PhD}$ theses.

KEY WORDS: theoretical frameworks - objects of study - methodology - objectives - hypothesis - techniques - communication research - UNAM - theses.

\section{DIAGNÓSTICO DA CONSTRUÇÃO TEÓRICA DE INVESTIGAÇÕES EM COMUNICAÇÃO NO MÉXICO}

\section{RESUME}

O proposito deste artigo é conhecer o estado atual que guarda a construção teórica de investigações em comunicação no México, mediante a identificação e analises de objetos de estudo e teorias que se manejam em 90 teses do programa de Pósgraduação em Ciências Políticas e Sociais da UNAM, com orientação em Comunicação elaboradas desde 2012 ao dia de hoje e publicadas no sistema TESIUNAM. Se trata de um estudo quantitativo que desenvolve uma analises a partir de 16 reativos, para estabelecer as incidências de linhas de investigação e temas; elementos de desenho de investigação; referencias teóricas, autores e obras de referência; enfoques metodológicos e técnicas; tipos de resultados e grau de construção teórica original. Os dados obtidos enfatizam a dispersão de temas, a 
Gutiérrez Morales, I. M.; Aguilar Ramírez, L. A.; González Chaparro, I. L.; Pérez

Lima, A. M.; Sandoval Pantoja, M. G. y Suárez Bedolla, B. I.

Diagnóstico de la construcción teórica de investigaciones en Comunicación en México

preferência pelos enfoques qualitativos em dois de cada três teses realizadas e por determinados autores e obras de referência; assim como o baixo nível de crítica ou construção teórica original, ainda nas teses de nível de doutorado.

PALAVRAS CHAVE: referencias teóricas - objetos de estudos - metodologiaobjetivos - hipóteses - técnicas - investigação em comunicação - UNAM - teses.

\section{Como citar el artículo:}

Gutiérrez Morales, I. M.; Aguilar Ramírez, L. A.; González Chaparro, I. L.; Pérez Lima, A. M.; Sandoval Pantoja, M. G. y Suárez Bedolla, B. I. (2020). Diagnóstico de la construcción teórica de investigaciones en Comunicación en México. [Diagnosis of the theoretical construction of Communication research in Mexico]. Vivat Academia. Revista de Comunicación, 150, 25-46.

doi: http://doi.org/10.15178/va.2020.150.25-46

Recuperado de http://www.vivatacademia.net/index.php/vivat/article/view/1099

\section{INTRODUCCIÓN}

La comunicación, como campo de conocimiento, ha abrevado de saberes teóricos propios de otras disciplinas, que han permitido a los investigadores construir objetos de estudio complejos, cuyas significaciones, explicaciones y reflexiones ameritan un trabajo interdisciplinario. Dentro de estos saberes es posible apreciar una gama interesante de postulados que van desde las originarias teorías de los efectos, hasta las teorías de las hipermediaciones, pasando por el estudio de las formas simbólicas, los estudios del discurso, las representaciones sociales, la semiótica, las teorías críticas, las teorías de la organización social, entre otras, que se han adoptado para enriquecer la ciencia de la comunicación, desde la sociología, la lingüística, la psicología, la teoría política, etc.

A un siglo de inaugurar la comunicación como disciplina (producto de la fascinación por los medios), los objetos de estudio también se han diversificado y, en últimas fechas, también se consideran los procesos de comunicación interpersonal y grupal, así como las pautas y producciones culturales, objetos de investigación y focos de la investigación en comunicación.

La copiosa investigación en comunicación ha logrado consolidar una disciplina, dispersa en objetos de estudio, teorías, metodologías y técnicas, que responden -desde la interdisciplinariedad- a construir conocimiento sobre los procesos comunicativos. Sin embargo, es un pronunciamiento constante en obras que manifiestan esfuerzos por crear teorías de la comunicación, o en foros donde concurren investigadores del área, la necesidad de unificar el campo, promover un diálogo entre investigadores e impulsar una base teórica que se asuma como propia de la disciplina.

Dentro de la investigación, una vez construido un problema de estudio, la necesidad de acogerse a una corriente teórica establece un primer dilema intelectual 
Gutiérrez Morales, I. M.; Aguilar Ramírez, L. A.; González Chaparro, I. L.; Pérez

Lima, A. M.; Sandoval Pantoja, M. G. y Suárez Bedolla, B. I.

Diagnóstico de la construcción teórica de investigaciones en Comunicación en México

que los investigadores deben resolver: decantarse por una opción teórica que permita iluminar el aspecto esencial del problema de investigación y la manera en que habrá de visualizarse el fenómeno investigado. Como consecuencia, en la investigación en comunicación, la dispersión y el préstamo teórico son obligados, aunque no perjudiciales para el avance científico.

Aunado a esto, las condiciones contextuales, por supuesto, son esenciales para definir esta acogida teórica, pues el objeto de estudio siempre se verá incidido por un entorno anclado a coordenadas espacio-temporales concretas. Por eso, al hablar de teorías contemporáneas de la comunicación, es menester reconocer que un cambio en las condiciones contextuales hace más o menos favorable la emergencia, adaptación y adopción de determinadas teorías. Y que la elaboración de un mapa teórico de la comunicación hoy en día seguramente exhibirá diferencias con respecto a un mapa elaborado, por ejemplo, a mediados del siglo XX.

\section{OBJETIVOS}

Es justamente, esa idea de generar un mapa teórico de la comunicación, específico para el caso de México, la que incentiva la investigación que a continuación se plantea. Señalar tendencias en cuanto a objetos de estudio, teorías, autores y métodos en la investigación en comunicación puede coadyuvar a reconocer las preocupaciones y las estrategias de atención a los problemas comunicativos, sistematizar el conocimiento que se ha adquirido en el campo y aportar elementos para una unificación de los saberes propios de la disciplina.

\section{ESTADO DE LA CUESTIÓN}

De acuerdo con Raúl Fuentes Navarro (2011, p. 214) es a mediados de la década de 1970 que en México comienzan a registrarse actividades de investigación científica y sistemática en materia de comunicación. Esto habla de un campo joven y fértil que se ha venido institucionalizando con grandes esfuerzos desde las entidades académicas nacionales (Fuentes 2014, p. 43), las cuales desde entonces han propuesto modos distintos de estudiar la comunicación. Sin embargo, en palabras de Tanius Karam (2009, p. 71):

El campo académico de la comunicación es muy amplio y diverso; por otra parte, se caracteriza también por su desarticulación entre la enseñanza y la investigación, entre investigación y campos profesionales, entre éstos y la primera. Las tensiones entre campo profesional y académico son agudas.

Lo anterior se debe, por un lado, al corto tiempo de existencia del campo en el panorama nacional y, por otro, a una marcada temporización de los enfoques teóricometodológicos desde los que se han explorado los fenómenos en la disciplina. Tal y como lo menciona Campos (2009), en un primer momento los estudios en comunicación se nutrieron de los aportes norteamericanos en el estudio de los medios de difusión, tales como el radio y la televisión. 
Gutiérrez Morales, I. M.; Aguilar Ramírez, L. A.; González Chaparro, I. L.; Pérez

Lima, A. M.; Sandoval Pantoja, M. G. y Suárez Bedolla, B. I.

Diagnóstico de la construcción teórica de investigaciones en Comunicación en México

En un segundo momento, en la década de 1970, se registra la llegada de la perspectiva europea a México con propuestas teóricas tales como la teoría crítica y la semiótica. De acuerdo con Karam (2009), es en este momento cuando las escuelas de comunicación apuestan por darle un mayor peso teórico a los planes y programas, superando la formación de personal técnico, pero impidiendo que "los egresados fueran capaces de realizar diagnósticos y evaluar situaciones concretas, ni mucho menos planificar en comunicación" (Karam, 2009, p. 75).

Hoy en día, tal y como lo menciona González (2015), en la investigación en comunicación "se establece una mayor amplitud teórica y metodológica", lo que obliga a los investigadores del campo a mirarse a sí mismos y a hacer un recuento de aquello que ha sido explorado, para así crear mapas que señalen los caminos andados $y$ aquellos otros que quedan vírgenes y que se establecen como oportunidades. Es así, que de acuerdo con la AMIC (citada por Campos, 2009) se aprecian 16 líneas de investigación en el territorio mexicano, de las cuales las siguientes se establecen como líneas de tradición: Comunicación Política, Economía Política de la Comunicación y la Cultura: Recepción de Medios; Estudios de Periodismo; Comunicación y Educación; Semiótica, discurso y lenguaje; Género y Comunicación; Políticas de Comunicación; Estudios sobre Medios (radio, cine y televisión). De más reciente exploración se encuentran las líneas de Sociedad de Información; Internet, Cibercultura y Telecomunicaciones; Comunicación Integral en las Organizaciones; Historia de la Comunicación; Comunicación Intercultural; Sociedad civil, Participación y Comunicación; Estudios de Juventud y Comunicación; y Comunicación Intersubjetiva.

En este sentido, Fuentes Navarro (2014, p. 44), con su extenso y profundo conocimiento sobre la evolución del campo y sus investigaciones, considera que:

Cada vez es más difícil identificar los estudios de comunicación con las investigaciones sobre medios (...) aunque se siguen haciendo trabajos estructurales y coyunturales sobre las industrias, hay mucha mayor especificidad que antes en cuanto a los aspectos considerados políticos, económicos, ideológicos, laborales, tecnológicos y una vinculación más concreta de estos aspectos con los factores contextuales.

El autor considera que este mismo fenómeno también es notable en las investigaciones de corte cultural, las cuales han cedido a "fuertes tendencias a economizarlas, politizarlas y tecnologizarlas, es decir, a diversificar y profundizar más específicamente las dimensiones socioculturales de objetos genéricos" (Fuentes, 2014, p. 44). Este fenómeno puede explicarse, según los hallazgos de dicho autor, gracias a que cada vez más los investigadores incorporan contenido empírico a su trabajo, lo cual les exige focalizar los estudios.

Tal y como se había mencionado al principio de este apartado, la investigación en comunicación ha logrado institucionalizarse gracias al esfuerzo de las universidades con sus programas de licenciatura y posgrado; en este respecto, Fuentes Navarro (2014, p. 44) expone que entre 1986 y 1994, el 20\% de las tesis presentadas abordaban 
Gutiérrez Morales, I. M.; Aguilar Ramírez, L. A.; González Chaparro, I. L.; Pérez

Lima, A. M.; Sandoval Pantoja, M. G. y Suárez Bedolla, B. I.

Diagnóstico de la construcción teórica de investigaciones en Comunicación en México

el tema de los medios y que la gran mayoría de los trabajos de este periodo "son más bien trabajos de investigación en ciencias sociales o en educación que en comunicación".

En el caso específico de los estudios sobre la investigación generada al interior de los programas de posgrado en Comunicación, resalta el esfuerzo de María Susana González Reyna (2015), quien señala que durante el periodo 2000-2015 el campo de investigación más trabajado en la UNAM es el de Comunicación y Cultura, la cual aglutina el $58 \%$ de las tesis de grado y cuya línea de investigación principal es "Comunicación, arte, lenguaje y sociedad". El segundo campo más explorado es Comunicación Política y Opinión Pública con el 21.5\% de los trabajos de grado y cuya línea de investigación principal es "Comunicación, opinión pública y cultura política". Finalmente, el campo con menos producción de trabajos de grado es el de Comunicación e Innovaciones Tecnológicas con el 20.5\% de los trabajos de grado y cuya línea principal es "Innovaciones tecnológicas y sociedad".

De acuerdo con la autora (González, 2015 p. 3152), estos trabajos de investigación dan lugar a "nuevas reflexiones teóricas que acuñan conceptos; asimismo, se aplican desde nuevas perspectivas, los métodos y las técnicas de investigación clásicas cuantitativas y cualitativas".

En resumen, el campo de la comunicación, con la juventud que le caracteriza, cobija dentro de sí el estudio de temáticas y enfoques diversos. La posibilidad de que alcance su madurez parece depender de la capacidad de los investigadores para poder entender y poner en perspectiva esta multiplicidad de caminos de exploración. En este sentido, Karam (2009, p. 421) afirma que uno de los retos por alcanzar es el de la "sistematización de información y establecimiento de bases de datos que permitan la realización de estados del arte más efectivos", es así que trabajos como el presente son un grano de arena más para alcanzar esta meta.

\section{METODOLOGÍA}

\subsection{Marco conceptual}

Un punto esencial de esta investigación fue elaborar un mínimo marco teórico que ayudara a entender el papel de la teoría en las investigaciones en comunicación. Esto remite a preguntarse sobre el sentido de la teoría, del conocimiento científico y de la epistemología disciplinaria. En otras palabras, ¿por qué es importante elaborar marcos teóricos? y, fundamentalmente, ¿por qué es importante la teoría para el avance científico? El propósito de esto es buscar el sustento pertinente para entender qué es la teoría y poder identificarla en el corpus de investigación.

En ese sentido, se entiende que la investigación es una práctica creativa y reflexiva, que tiene su base en la capacidad del ser humano de hacerse preguntas sobre sí mismo y sobre la realidad, desde una perspectiva específica y en una 
Gutiérrez Morales, I. M.; Aguilar Ramírez, L. A.; González Chaparro, I. L.; Pérez

Lima, A. M.; Sandoval Pantoja, M. G. y Suárez Bedolla, B. I.

Diagnóstico de la construcción teórica de investigaciones en Comunicación en México

búsqueda por obtener respuestas que le permitan encarar el mundo e incorporarse a él (Juárez, 1997, p. 23).

Así sobrevivieron los primeros seres humanos en un mundo en el cual se encontraban en franca desventaja frente a otras especies y a las inclemencias de su hábitat. La práctica reflexiva también permitió que se configuraran las cosmogonías y estructuras sociales de las primeras grandes civilizaciones. Investigar era una actividad fundamentalmente ontológica y axiológica, preocupada por el quién y el para qué.

Sin embargo, el mundo devino cada vez más complejo y con ello, las preguntas y sus explicaciones requirieron de una actividad heurística más estructurada, exigida de asentarse sobre un camino perfectamente estructurado y con un sustento teórico. Apareció la ciencia como un conjunto de conocimientos racionales, ciertos o probables, obtenidos de manera metódica y verificados en su contrastación con la realidad (Ander Egg, 2004).

Investigar se convirtió en una actividad con estatus esencialmente científico; toda respuesta o explicación válida ya no podía ser solamente ontológica y axiológica, sino rigurosamente metodológica y epistemológica. La teoría, entonces, tomó un papel primordial.

Con frecuencia, los que se inician en la investigación no advierten la importancia de una teoría. La falta de explicitación de la misma hace perder eficacia y coherencia al proceso de investigación; desde el diseño metodológico hasta la interpretación de datos y finalmente, la enunciación de conclusiones.

En el contexto de la investigación científica, una teoría es un sistema de ideas, un marco de interpretación que el científico crea o recurre para dar una explicación a hechos, datos o fenómenos. Implica una serie de juicios, argumentaciones o proposiciones interpretativas a partir de las cuales se mira la realidad y se problematiza (Schlanger, 1983).

Como toda interpretación o creación humana, una teoría es hipotética o supuesta, y finalmente puede ser verdadera o falsa. Sin embargo, según Einstein (1981, p. 44):

Las experiencias sensoriales representan lo dado. Pero la teoría que tendrá que interpretarlas está hecha por el hombre. Se trata del resultado de un proceso de adaptación de carácter extremadamente arduo: hipotético, nunca definitivo, siempre sujeto a la crítica y a la duda.

La manera científica de formar conceptos se distingue de la que utilizamos en la vida de cada día no sustancialmente, sino sólo en la mayor precisión de las definiciones de los conceptos y las conclusiones; una elección más esmerada y sistemática del material experimental; una mayor economía lógica. Esto último significa el esfuerzo por reducir todos los conceptos y axiomas básicos lógicamente independientes. 
Gutiérrez Morales, I. M.; Aguilar Ramírez, L. A.; González Chaparro, I. L.; Pérez

Lima, A. M.; Sandoval Pantoja, M. G. y Suárez Bedolla, B. I.

Diagnóstico de la construcción teórica de investigaciones en Comunicación en México

En este sentido, Popper (1994) pone de manifiesto que una teoría como construcción de una interpretación hipotética de la realidad: a) admite la existencia de problemas porque existen contradicciones, entre las ideas o entre las ideas y la realidad; b) implica discernir o explicar dichas contradicciones a través de un método lógico y sistemático; y c) la creación de una idea o sistema de ideas, con diversos grados de abstracción o generalidad, con la cual se explican o excluyen las contradicciones.

Finalmente, toda teoría será más sólida en la medida en que tenga una base rigurosa de construcción y que pueda cumplir los siguientes criterios:

1. Ser un sistema de ideas o una serie de proposiciones que están ordenados lógicamente, con coherencia y sin contradicciones, partiendo de los más abstractos o generales.

2. Ser amplia o fecunda. Sus conclusiones deben explicar todos los casos problemáticos conocidos en un campo científico, y sugerir nuevas explicaciones, más allá de lo observado o explicado hasta el momento.

3. Ser simple. La teoría que con menos supuestos explica más consecuencias es mejor que la que requiere más supuestos para explicar lo mismo.

4. Ser comunicable. Este elemento lo propone Mario Bunge (1993, p. 14-15), quien refiere que la comunicabilidad es posible gracias a la precisión; y es a su vez una condición necesaria para la verificación de los datos empíricos y de las hipótesis. La comunicación de los resultados y de las técnicas de la ciencia no sólo perfecciona la educación general, sino que multiplica las posibilidades de su confirmación o refutación.

\subsection{Conformación del corpus}

El corpus de esta investigación quedó conformado por tesis de grado (maestría y doctorado), elaboradas por alumnos del Programa de Posgrado en Ciencias Políticas y Sociales de la Universidad Nacional Autónoma de México. Las tesis, no sólo constituyen un mecanismo para alcanzar un título o una certificación oficial para el desempeño profesional de un individuo, sino que obligan a su autor a incursionar en la disciplina en la que se forma y atender problemas relativos a su campo mediante la investigación científica (básica o aplicada). Se parte de la idea de que un trabajo de tesis es una investigación que abona al saber disciplinario, y que debe observar rigor metodológico y aportar conocimiento original. Las tesis de grado implican mayor nivel de profundización en la disciplina, ya que deben revestirse de saber especializado, y ya no genérico del campo (nivel licenciatura); además constituyen la base de un posible trabajo académico que los investigadores realizan para incursionar -como especialistas- en una línea concreta. Tienen mayores posibilidades de difusión en foros disciplinarios y también mayores posibilidades de seguimiento y profundización por parte de sus autores.

La selección de este acervo textual obedece, no sólo a la accesibilidad que permiten las bases de datos y redes de la Universidad Nacional Autónoma de México, a través de TESIUNAM, sino a que la UNAM y, en particular, el Programa de Posgrado en 
Gutiérrez Morales, I. M.; Aguilar Ramírez, L. A.; González Chaparro, I. L.; Pérez

Lima, A. M.; Sandoval Pantoja, M. G. y Suárez Bedolla, B. I.

Diagnóstico de la construcción teórica de investigaciones en Comunicación en México

Ciencias Políticas y Sociales siguen siendo un referente en la formación de científicos y tecnólogos y un semillero destacado de profesionales de la comunicación.

Las tesis seleccionadas se ciñeron a los siguientes criterios:

1. Ser producto de trabajos de investigación en el área de Comunicación.

2. Haberse realizado con el propósito de obtener los grados académicos de maestría o doctorado.

3. Haber sido elaboradas (publicadas) del año 2012 a marzo de 2017. Lo anterior para otorgar el estatus de "actual" a la construcción teórica de sus objetos de estudio.

En el sistema se encontraron registradas un total de 92 tesis de grado: 66 de maestría y 26 tesis doctorales; sin embargo, dos de estos trabajos no se encuentran disponibles para consulta en línea, lo que dio lugar a una muestra de 90 tesis: 65 de maestría y 25 de doctorado

\subsection{Diseño del instrumento de análisis}

El instrumento de análisis se diseñó a partir de 16 reactivos que se explican a continuación:

1. Línea de investigación. Reactivo de opción múltiple que incluyó como respuesta las 20 líneas definidas por la UNAM para realizar investigaciones de posgrado en el área de Comunicación, agrupadas en tres campos: Comunicación e Innovaciones Tecnológicas, Comunicación Política y Comunicación y Cultura.

2. Tema. Se trata de un reactivo de selección de una lista, que tuvo la finalidad de especificar los temas abordados, para identificar y detallar de manera concreta la orientación de la investigación.

Este reactivo ofreció las siguientes opciones de respuesta: Representaciones sociales; Legislación de los medios y derechos de autor; Análisis del discurso; Ética de los medios; Análisis de mensajes; Consumo cultural; Ética periodística; Formación profesional en comunicación; Análisis semiótico; Teorías y metodologías de la comunicación; Comunicación política; Literatura; Mediaciones sociales; Comunicación organizacional; Reconfiguración de la estructura multimediática; TIC y grupos sociales; Multiculturalidad, interculturalidad; Lectura; Análisis de contenido; Redes sociales y nuevos medios; Comunicación para el desarrollo; Periodismo; Grupos mediáticos y convergencia digital; Comunicación y sociedad; Comunicación, opinión pública y política; Cultura digital; Prácticas comunicativas y Usos sociales de los medios.

Para la clasificación de temas se utilizó la propuesta realizada por José Antonio Meyer Rodríguez y María Belén Fernández Fuentes (2012, p. 32). A diferencia de la línea de investigación, se propuso este reactivo para realizar especificaciones de las temáticas, en virtud de acercarse a los objetos de estudio construidos en cada tesis.

3. Objeto de estudio. Reactivo de opción múltiple que incluyó como respuesta los siguientes conceptos: Actores, Instrumentos, Expresiones (mensajes), Representaciones y Otros. 
Gutiérrez Morales, I. M.; Aguilar Ramírez, L. A.; González Chaparro, I. L.; Pérez

Lima, A. M.; Sandoval Pantoja, M. G. y Suárez Bedolla, B. I.

Diagnóstico de la construcción teórica de investigaciones en Comunicación en México

Para la clasificación de los objetos de estudio se seleccionó la tipología descrita por Manuel Martín Serrano (1982).

4. Ubicación espacial del objeto de estudio. Reactivo de opción múltiple que incluyó las siguientes opciones de respuesta: Macrosocial internacional, Macrosocial nacional, Microsocial internacional y Microsocial nacional.

La finalidad de este reactivo fue determinar la extensión en términos de tamaño de la comunidad estudiada (micro o macro social) y el alcance espacial (nacional o internacional) de las investigaciones.

5. Ubicación temporal del objeto de estudio. Reactivo de opción múltiple que ofrece las siguientes opciones de respuestas: Siglo XXI, Siglo XX, Siglo XIX y Anteriores.

El objetivo de este apartado fue identificar la época o períodos específicos en los que se ubica el objeto de estudio investigado.

6. Objetivos. Es una pregunta de tipo binario que da como opciones de respuesta

Sí o No. La finalidad de este reactivo fue identificar la inclusión explícita en las tesis del o de los objetivos de la investigación.

La importancia de este reactivo radica en que como lo señalan las normas APA (American Psychological Association, s.f.): los objetivos de un trabajo de grado son una aspiración de lo que se espera conseguir o demostrar; ofrecen una descripción sucinta de la orientación y rumbo del trabajo, por tanto, son guías de la investigación o pasos que se han dilucidado como los indicados para estudiar el problema de investigación y avalar la postura intelectual del tesista.

7. Hipótesis. Es un reactivo de tipo binario que da como opciones Sí o No. Con este reactivo se indagó en las tesis la presencia o no de la enunciación de hipótesis.

Es trascendental la presencia de hipótesis en las tesis ya que según las normas APA (American Psychological Association, s.f.) son suposiciones o predicciones que se hacen sobre los resultados de la investigación. Se consideran guías que nos permiten orientar el trabajo a la consecución de un objetivo o conclusión determinada.

8. Marco teórico. Es un reactivo de carácter abierto que busca identificar cuál o cuáles son las teorías, establecidas previamente, de las que se vale el autor para desarrollar su investigación.

9. Marco conceptual. Reactivo de carácter abierto que busca identificar cuál o cuáles son los conceptos que el autor desarrolla como base de su disertación.

10. Autores para la construcción teórica. Reactivo de carácter abierto que busca identificar cuál o cuáles son los autores a los que recurre el autor de la tesis para el modelado del objeto de estudio de la investigación.

11. Obras para la construcción teórica del objeto de estudio. Reactivo de carácter abierto que busca identificar cuál o cuáles son las obras a las que recurre el autor de la tesis para el modelado del objeto de estudio de la investigación.

12. Tipo de metodología. Reactivo de opción múltiple que ofrece las siguientes opciones de respuesta: cualitativa, cuantitativa y mixta. Se entiende por metodología cualitativa a la que recurre al análisis de las características y cualidades del objeto de estudio a diferencia de la cuantitativa que recurre al análisis numérico de datos relacionados con el mismo objeto. Una 
Gutiérrez Morales, I. M.; Aguilar Ramírez, L. A.; González Chaparro, I. L.; Pérez

Lima, A. M.; Sandoval Pantoja, M. G. y Suárez Bedolla, B. I.

Diagnóstico de la construcción teórica de investigaciones en Comunicación en México

metodología mixta se entiende como la combinación de análisis de tipo cuantitativo y cualitativo.

13. Tipo de investigación. Es un reactivo de tipo binario que ofrece como opciones de respuesta básica y aplicada. Se considera a una investigación básica a la que sus resultados abonan a la comprensión de un fenómeno u objeto de estudio, así como alguna aportación teórica. Por otro lado, la investigación se considera aplicada cuando resulta en un producto determinado como una guía, procedimiento, material didáctico o de cualquier otra naturaleza.

14. Técnicas empleadas. Se trata de un reactivo de selección de una lista que refiere a las técnicas de investigación empleadas en los trabajos de tesis y que ofrece las siguientes opciones de respuesta: análisis documental (comentario a bibliografía diversa), análisis del discurso, análisis de contenido, análisis semiótico, análisis de mensajes (diferente del discursivo, de contenido o semiótico), entrevista, entrevista a profundidad, encuesta, grupos de discusión, grupos focales, técnicas proyectivas, observación participante, observación no participante, técnicas sociométricas, análisis prospectivo, historias de vida, bitácora o diario de campo y otras.

15. Resultados. Es una pregunta de tipo binario que ofrece como posibilidades de respuesta cuantitativos y cualitativos. Se comprende por resultados cuantitativos a los que son de carácter numérico a partir del análisis estadístico de datos y cualitativos a los que son de carácter descriptivo a partir del análisis de las características y cualidades del objeto de estudio.

16. Construcción teórica en las conclusiones. Es una pregunta de tipo binario que da como opciones sí o no. Refiere si el autor de la investigación integra aportaciones teóricas en las conclusiones de la investigación, ya sea para elaborar críticas de sus bases teóricas o planteamientos teóricos originales.

\section{RESULTADOS}

\subsection{Línea de investigación}

Las tres líneas de investigación que agrupan la mayor cantidad de investigaciones en el posgrado son: a) Comunicación y sociedad; b) Estudios de cine; y c) Estudios sobre periodismo. Si agrupamos estos datos de acuerdo a los tres campos que ofrece el Programa de Posgrado en Ciencias Políticas y Sociales, concordamos con los resultados obtenidos por González (2015), en cuanto a que el campo de Comunicación y Cultura es el que más trabajos reúne con un $65.5 \%$ de las tesis realizadas en los últimos cinco años. El 18.8\% de las tesis corresponden al campo de Comunicación política; y el 13.3\%, al de Comunicación e innovaciones tecnológicas.

Cabe señalar que se agregó una línea de investigación, que se nombró "Epistemología de la comunicación" y que refiere trabajos que hacen críticas o propuestas teóricas a propósito de la comunicación. En esta línea se identificaron dos trabajos (ambos de nivel doctorado), lo que corresponde al 2.2\% del corpus total. 
Gutiérrez Morales, I. M.; Aguilar Ramírez, L. A.; González Chaparro, I. L.; Pérez Lima, A. M.; Sandoval Pantoja, M. G. y Suárez Bedolla, B. I.

Diagnóstico de la construcción teórica de investigaciones en Comunicación en México

\subsection{Tema}

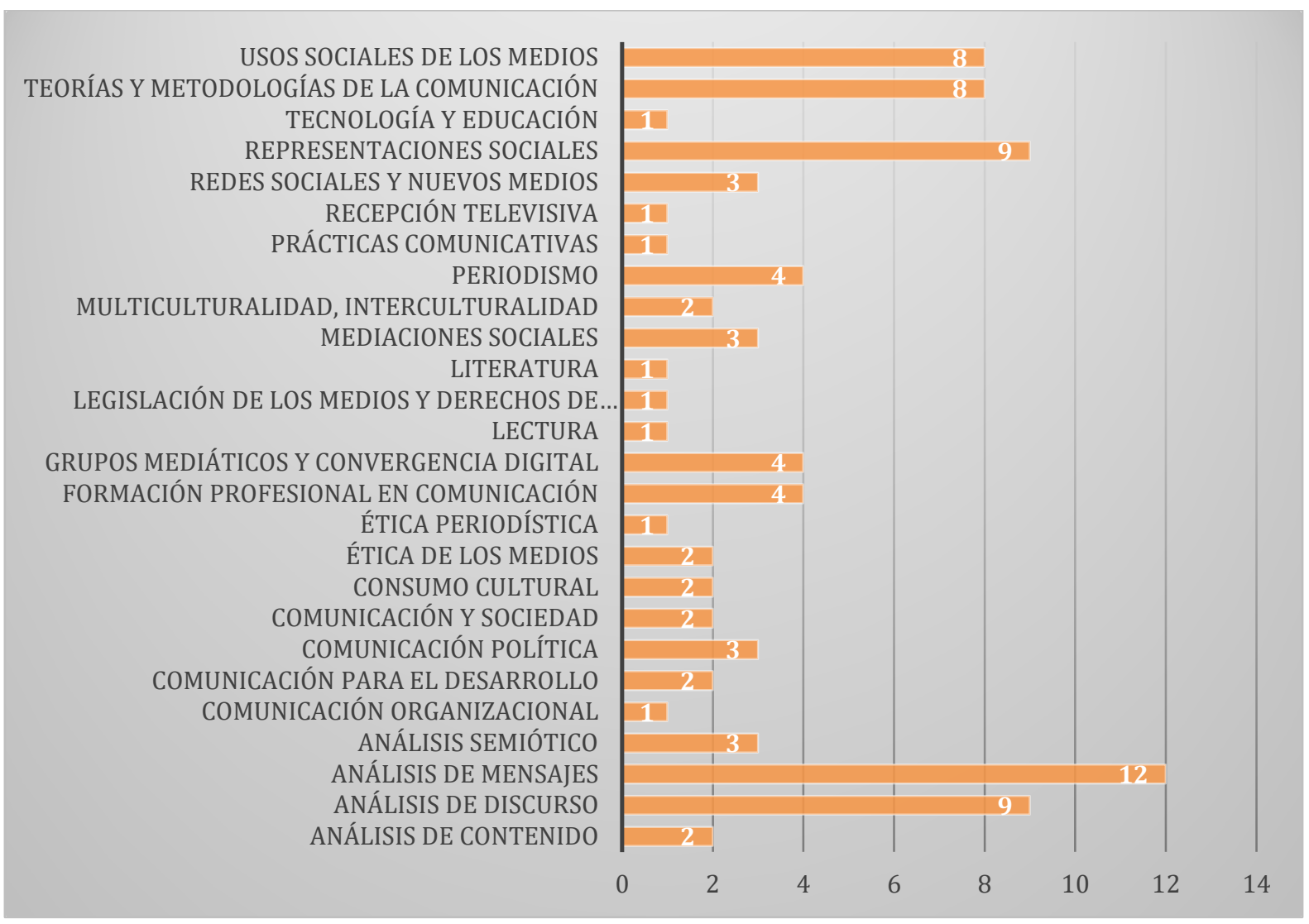

Gráfico 1. Frecuencia de temas.

Fuente: elaboración propia.

El gráfico 1 muestra una dispersión importante de las temáticas abordadas en las investigaciones de grado en materia de comunicación. El análisis de mensajes, el análisis del discurso, las representaciones sociales, los usos sociales de los medios y las teorías y metodologías de la comunicación constituyen los temas específicos de más de la mitad de las tesis (51.1\%). Los temas restantes, de manera individual, no alcanzan el $5 \%$ de representatividad.

\subsection{Objetos de estudio}

Para este reactivo se estableció una tipología que permitió simplificar la versatilidad de objetos de estudio a los que puede recurrir un investigador de la comunicación. Al definir los elementos de la comunicación, según el modelo dialógico de Martín Serrano (1982), dicho autor establece cuatro elementos fundamentales: los actores, los instrumentos, las expresiones y las representaciones.

Retomando dicha tipología, el objeto de estudio más recurrido por los investigadores son las expresiones, es decir, los mensajes de la comunicación. Adicionalmente, si se recupera el gráfico 1 sobre la clasificación temática, se 
Gutiérrez Morales, I. M.; Aguilar Ramírez, L. A.; González Chaparro, I. L.; Pérez Lima, A. M.; Sandoval Pantoja, M. G. y Suárez Bedolla, B. I.

Diagnóstico de la construcción teórica de investigaciones en Comunicación en México observará que, en efecto, la preocupación prioritaria de la investigación en comunicación en la UNAM muestra un pronunciado acento hacia el estudio del contenido de los mensajes en los procesos comunicativos.

De los cuatro elementos definidos por Martín Serrano, los actores constituyen el objeto de estudio tan sólo en el $11.1 \%$ de los trabajos de tesis. Mientras que los instrumentos (entendidos fundamentalmente como los medios de comunicación) y las representaciones (dentro de las que encontramos significados, opiniones y otros objetos ideales) se estudian cada uno en el 16.6\% de las investigaciones. Asimismo, en 14 tesis $(15.5 \%)$, aparecieron distintas combinaciones de los ítems mencionados con anterioridad.

\subsection{Ubicación espacial}

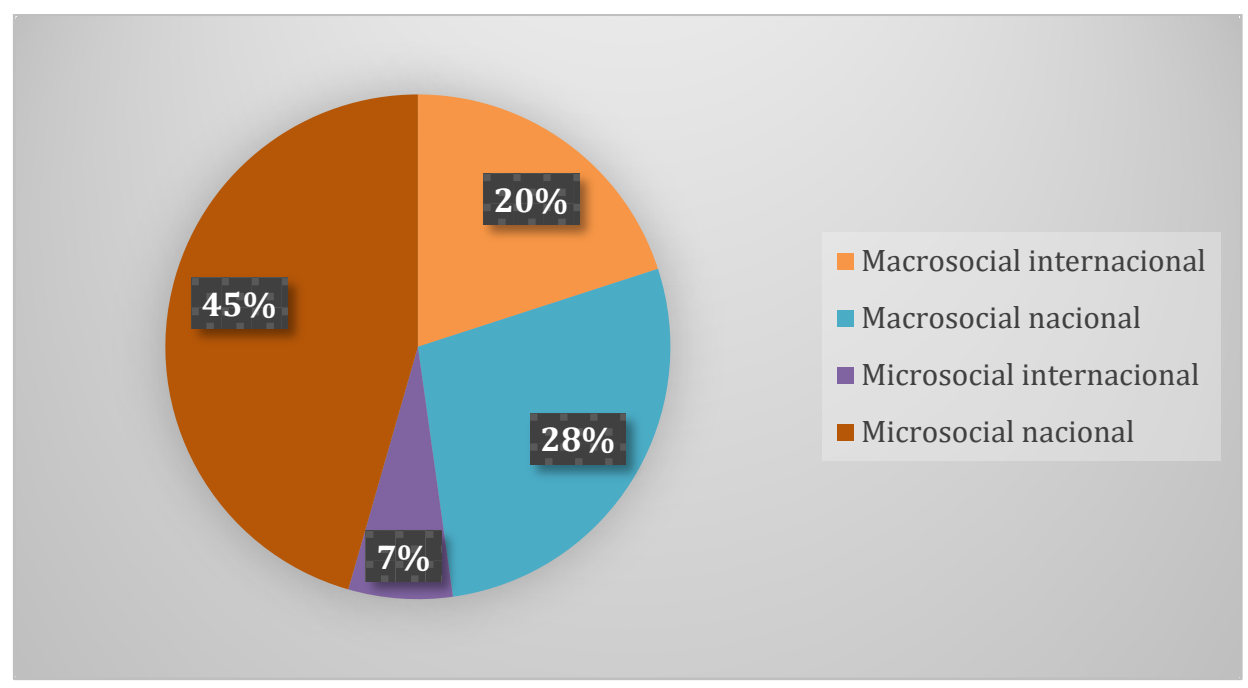

Gráfico 2. Ubicación espacial.

Fuente: elaboración propia.

La investigación en comunicación que se realiza en el Programa de Posgrado de la UNAM es mayoritariamente local. El alcance microsocial nacional nos habla del estudio de poblaciones específicas, de comunidades focalizadas territorialmente, quizás universos poblacionales a nivel sectorial o municipal. Si unimos los porcentajes de micro y macrosocial a nivel nacional, encontraremos que el $73 \%$ de las investigaciones en comunicación se basan en el estudio de problemáticas mexicanas, y que sólo el $27 \%$ torna la mirada hacia el contexto internacional.

\subsection{Ubicación temporal}

El porcentaje mayoritario de las investigaciones en comunicación ubica temporalmente sus objetos de estudios en el presente siglo XXI (88\%). Aunque el siglo XX todavía sigue teniendo presencia en las tesis analizadas, el porcentaje de aparición asciende tan solo al 11\%. Mientras que solo una tesis ubica su objeto de estudio en el siglo XIX. Esto habla de la construcción de problemas de estudio 
Gutiérrez Morales, I. M.; Aguilar Ramírez, L. A.; González Chaparro, I. L.; Pérez Lima, A. M.; Sandoval Pantoja, M. G. y Suárez Bedolla, B. I.

Diagnóstico de la construcción teórica de investigaciones en Comunicación en México actuales, vigentes $\mathrm{y}$, si se combina con los resultados de la gráfica (d), estamos ante investigaciones que acusan un grado importante de proximidad espacio-temporal de los objetos de estudio.

\subsection{Objetivos}

El señalamiento explícito de los objetivos de investigación está presente en el $84 \%$ de las tesis analizadas. Sin embargo, el hecho de que en el 16\% de las mismas no aparezcan podría señalar debilidades en el diseño de las investigaciones.

\subsection{Hipótesis}

Caso distinto al de los objetivos es el de la enunciación de las hipótesis de las investigaciones. Aunque aparecen en el porcentaje mayoritario de las tesis (64\%), el hecho de que casi 4 de cada 10 tesis no haga explícitas las hipótesis habla de una falta de orientación de los resultados esperados en la etapa del diseño del proyecto.

\subsection{Marcos teóricos}

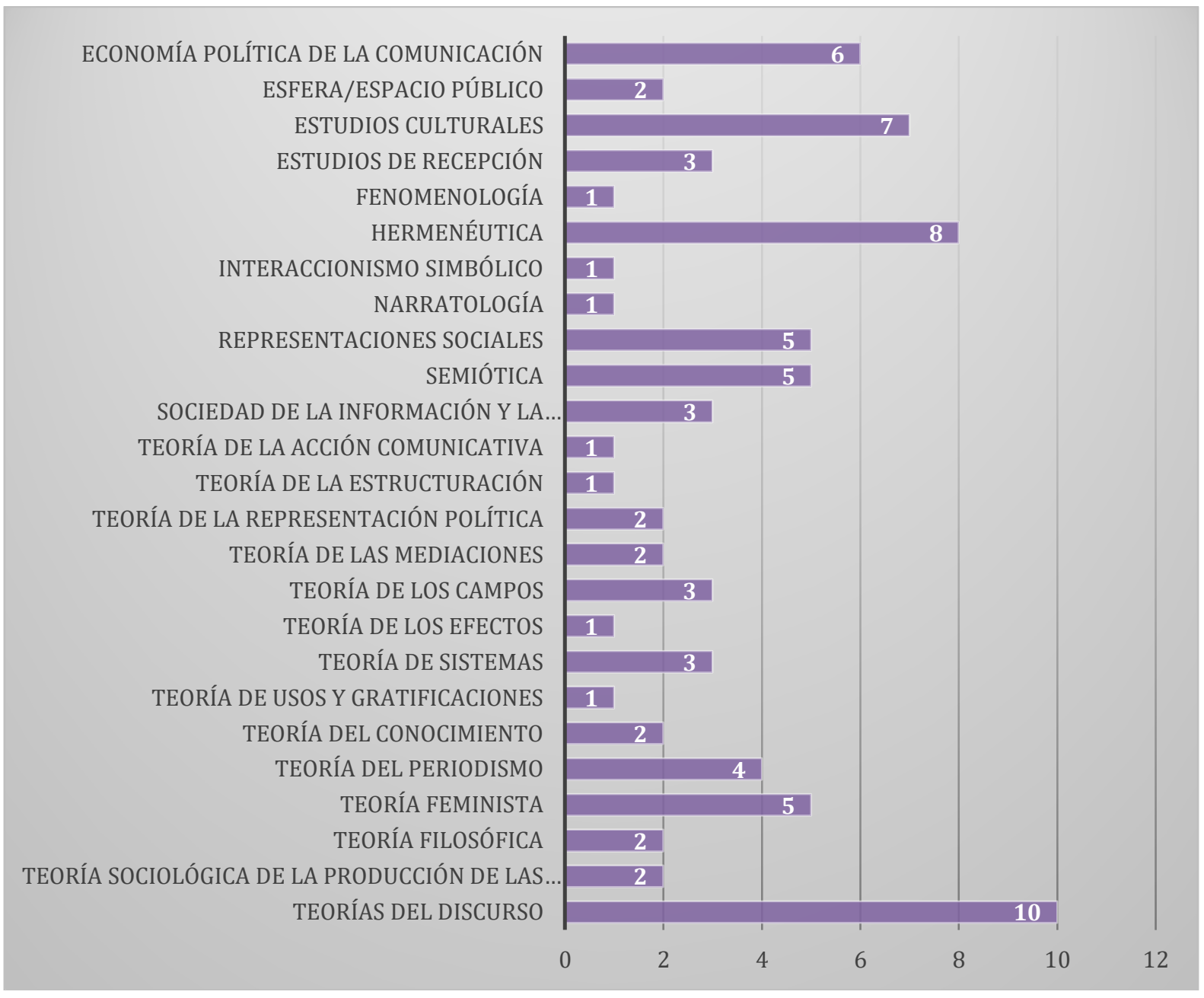

Gráfico 3. Teorías empleadas.

Fuente: elaboración propia. 
Gutiérrez Morales, I. M.; Aguilar Ramírez, L. A.; González Chaparro, I. L.; Pérez Lima, A. M.; Sandoval Pantoja, M. G. y Suárez Bedolla, B. I.

Diagnóstico de la construcción teórica de investigaciones en Comunicación en México

Las teorías más socorridas por los investigadores en comunicación subrayan la importancia del campo de la comunicación y la cultura. El acento prioritario se encuentra en las teorías del discurso, junto con la hermenéutica, los estudios culturales y la economía política de la comunicación. Otros enfoques teóricos destacados son los que corresponden a las representaciones sociales, la semiótica y la teoría feminista, como base de un número importante de las investigaciones que se realizan en el posgrado.

Es importante señalar que si se hace una revisión de los postulados teóricos que nutren las investigaciones en la UNAM, se encontrará un fuerte apego a las tradiciones consolidadas en el Programa de Posgrado y al saber de la comunicación en su devenir como disciplina científica. Es decir, se trata, en su mayoría, de teorías que provienen de otros campos disciplinarios que se adoptan para el estudio de la comunicación y nutren el ejercicio interdisciplinario en esta área.

\subsection{Marcos conceptuales}

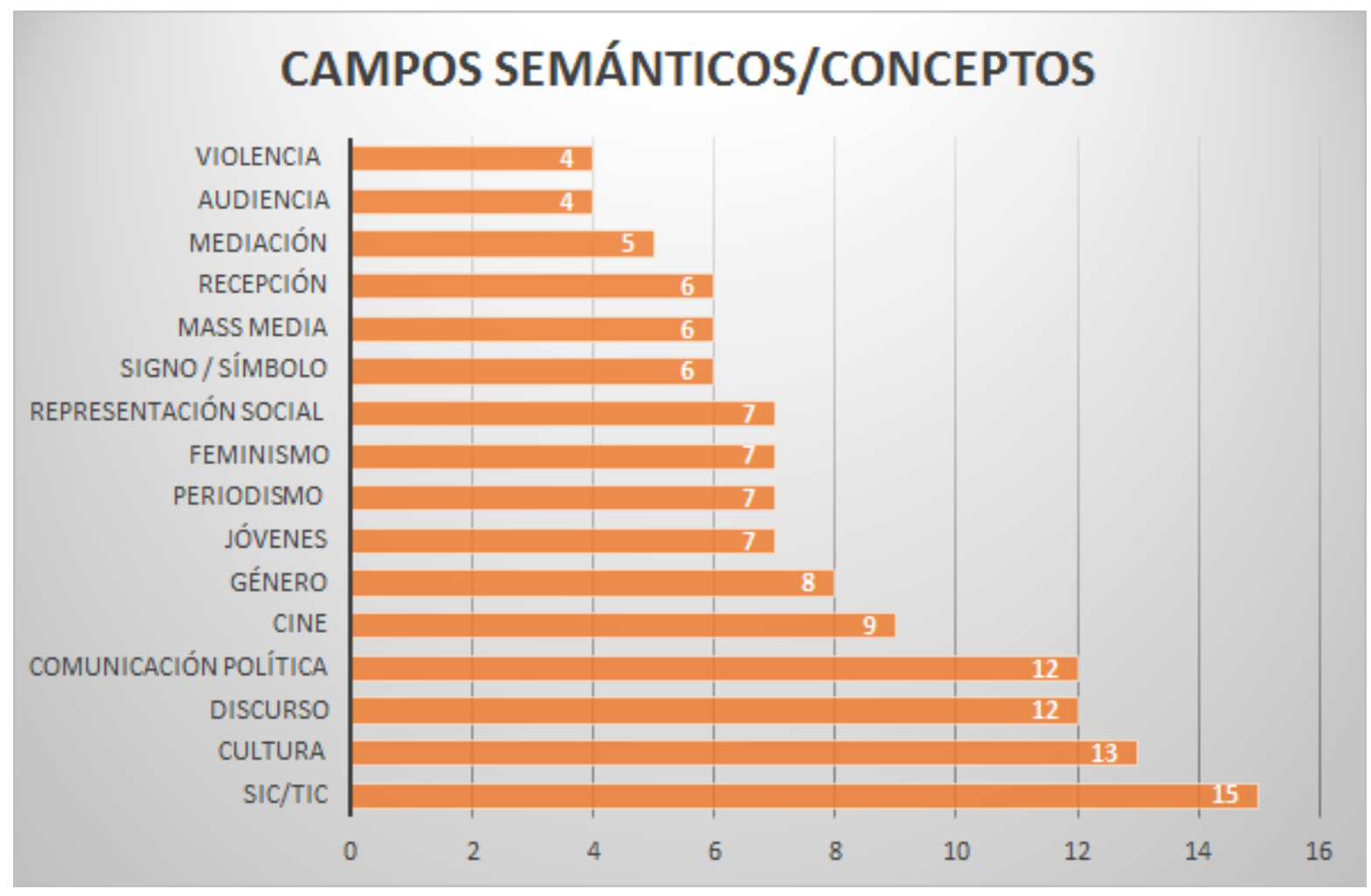

Gráfico 4. Campos semánticos/conceptos más desarrollados.

Fuente: elaboración propia.

Para el caso de este reactivo, se recuperaron todos los conceptos que los investigadores definen como base de la construcción teórica de sus objetos de estudio. Ante la numerosidad de dichos conceptos, se realizó un segundo ejercicio de clasificación por campos semánticos. Palmer (1978), Ullmann (1991) y Lyons (1997) coinciden en señalar que la teoría de los campos semánticos es una creación de Trier, 
Gutiérrez Morales, I. M.; Aguilar Ramírez, L. A.; González Chaparro, I. L.; Pérez

Lima, A. M.; Sandoval Pantoja, M. G. y Suárez Bedolla, B. I.

Diagnóstico de la construcción teórica de investigaciones en Comunicación en México

quien propuso la categoría de campo semántico o conceptual, entendiendo por ello un campo o un sector compuesto por una serie de elementos léxicos con relaciones recíprocas, en el cual cada elemento contribuye a delimitar el sentido de sus vecinos y a la vez es delimitado por ellos. Los campos semánticos, en suma, permiten una organización más o menos rigurosa e ilustrativa de las redes de sentido que se construyen alrededor de unidades lingüísticas significativas.

Como se observa en el gráfico 4, los conceptos más definidos en las tesis analizadas son los que corresponden a los campos semánticos de las TIC, de la cultura, del discurso y de la comunicación política.

\subsection{Autores para la construcción teórica del objeto de estudio}

Revisando los autores más utilizados para la construcción teórica del objeto de estudio, vemos de nueva cuenta la aparición de autores consolidados en el campo de la comunicación en México. No siempre se trata de autores que se reconozcan como especialistas en comunicación, por el contrario, en la lista de autores que asciende a más de 260 (sólo para la construcción teórica de los objetos de estudio) aparecen por igual filósofos, antropólogos, politólogos, lingüistas o historiadores. El legado de dichos autores ha sido adoptado por los comunicólogos para asentar las bases teóricas de su disciplina, nuevamente de manera interdisciplinaria.

Destacan como autores Manuel Castells, Jürgen Habermas, Gilberto Giménez, Guillermo Orozco y John B. Thompson con los mayores porcentajes de aparición en las investigaciones. Otros autores como Roland Barthes, Clifford Geertz, Michel Foucault, Charles Sanders Peirce, Armand Mattelart, Michel Pecheüx, Julia Kristeva, Gerard Genette o Edgar Morin, también citados en varias ocasiones en el corpus.

La escasa aparición de autores latinoamericanos también es sintomática y hasta contradictoria con respecto a la construcción de los objetos de estudio.

\subsection{Obras para la construcción teórica del objeto de estudio}

Manuel Castells y Jürgen Habermas se mantienen como los autores más citados con dos obras distintas cada uno. De la autoría de Castells aparecen Comunicación y Poder y La era de la información en sus tres tomos; mientras que, de Habermas, las tesis citan frecuentemente Historia y crítica de la opinión pública y Teoría de la acción comunicativa. También entran en la lista Los cautiverios de las mujeres de Marcela Lagarde, para apoyar estudios de género y comunicación; De los medios a las mediaciones de Jesús Martín Barbero; La economía política de la comunicación, de Vincent Mosco; Televisión y audiencias de Guillermo Orozco; Curso de Lingüística General de Ferdinand de Saussure; e Ideología y cultura moderna de la autoría de John B. Thompson. Todas ellas, obras de referencia obligada para los estudiosos de la comunicación en México. 
Gutiérrez Morales, I. M.; Aguilar Ramírez, L. A.; González Chaparro, I. L.; Pérez Lima, A. M.; Sandoval Pantoja, M. G. y Suárez Bedolla, B. I.

Diagnóstico de la construcción teórica de investigaciones en Comunicación en México

\subsection{Tipo de investigación}

En cuanto al tipo de investigación, un porcentaje significativo (87\%) corresponde a investigaciones de tipo básico, aquí entendida como la investigación que tiene por objeto la generación del conocimiento para la comprensión de un aspecto de la realidad. Mientras tanto, solo el 13\% de las investigaciones tienen un giro aplicado; es decir, el desarrollo de la tesis tuvo como objetivo la generación de productos (modelos, programas, manuales, etc.) que permitan la actuación frente a una problemática específica, para su atención o solución.

\subsection{Metodología}

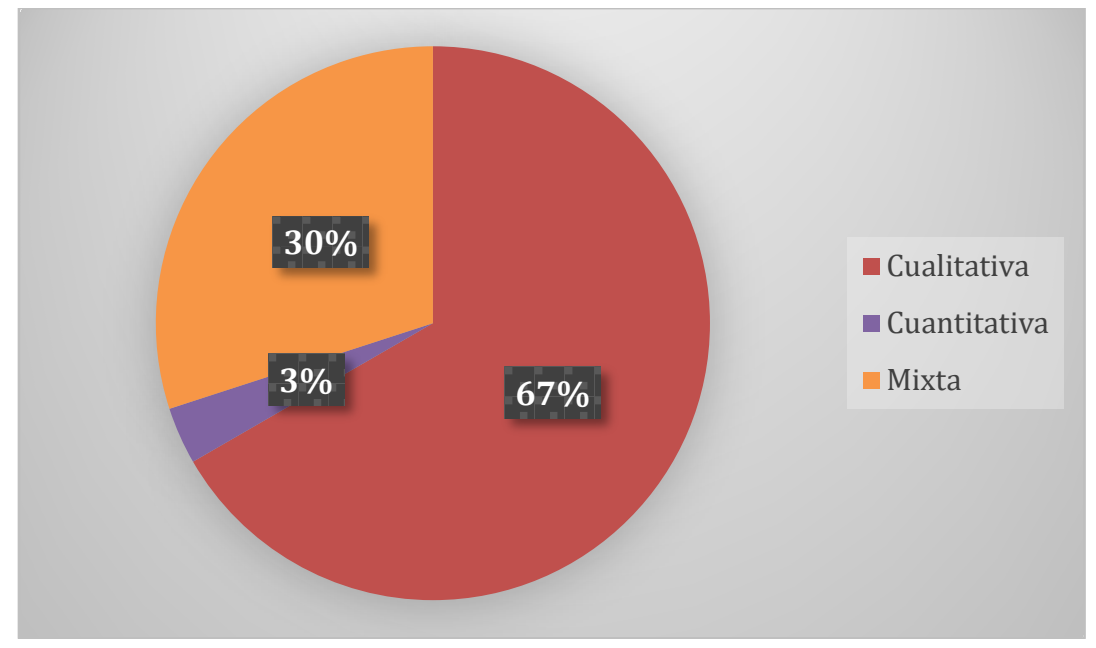

Gráfico 5. Tipo de metodología.

Fuente: elaboración propia.

El enfoque cualitativo aparece en casi 7 de cada 10 tesis analizadas. Esto marca la congruencia entre los estudios de comunicación como disciplina social, interesada más en procesos y significados atribuibles a sujetos sociales, que a datos numéricos y su tratamiento estadístico. 30\% de las investigaciones utilizan metodología mixta, y sólo el 3\% emplean una perspectiva puramente cuantitativa. 
Gutiérrez Morales, I. M.; Aguilar Ramírez, L. A.; González Chaparro, I. L.; Pérez Lima, A. M.; Sandoval Pantoja, M. G. y Suárez Bedolla, B. I.

Diagnóstico de la construcción teórica de investigaciones en Comunicación en México

\subsection{Técnicas}

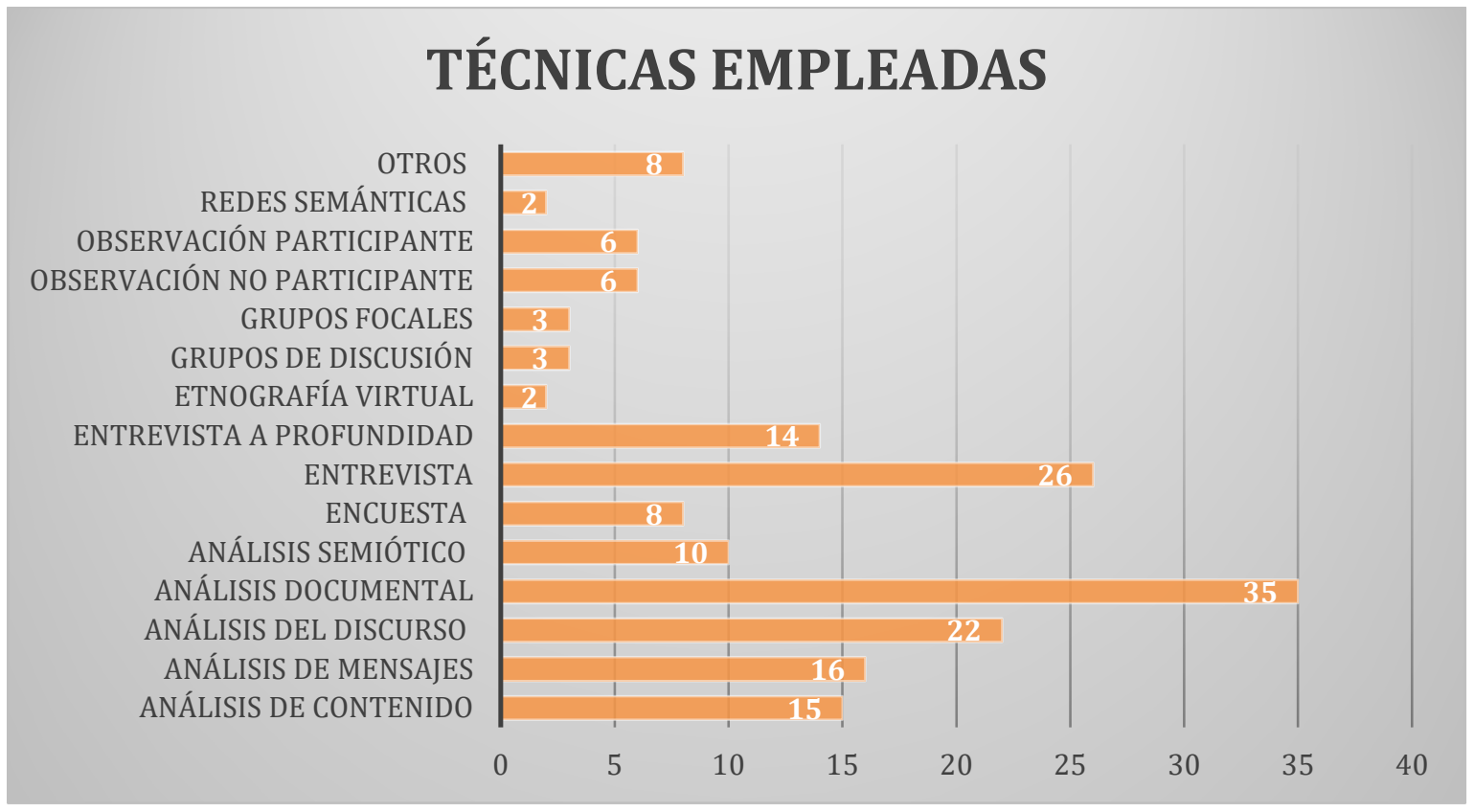

Gráfico 5. Técnicas más empleadas.

Fuente: elaboración propia.

El análisis documental, la entrevista y el análisis del discurso aparecen como las técnicas de investigación más utilizadas para la investigación en comunicación. Los investigadores también recurren al análisis de contenido, al análisis semiótico, a la observación, a la encuesta y a los grupos de discusión y focales. En el rubro 'Otros' aparecen con alguna mención las siguientes técnicas: el análisis crosesférico, el FODA, el análisis kinésico, la etnografía, el hipertexto reducido, la historia oral, el análisis de la representación y el análisis hermenéutico.

\subsection{Resultados}

En concordancia con el tipo de metodología empleada, los resultados cualitativos aparecen en el 69\% de las tesis analizadas; mientras el 28\% combina resultados de tipo cuantitativo y cualitativo y sólo el 3\% expone datos meramente numéricos.

\subsection{Construcción teórica en las conclusiones}

Únicamente tres de cada 10 tesis elaboran una crítica de las teorías empleadas para la construcción de los objetos de estudio, o bien, proponen líneas, postulados o parámetros para una posible categorización teórica novedosa de objetos de estudio de la comunicación. Esto nos habla de una falta de autonomía teórica en los estudiantes de posgrado, falta de profundización en sistemas teóricos o, incluso, cierto desdén para colaborar en la consolidación teórica del campo a partir de investigación sistemática referida al contexto actual mexicano. 
Gutiérrez Morales, I. M.; Aguilar Ramírez, L. A.; González Chaparro, I. L.; Pérez

Lima, A. M.; Sandoval Pantoja, M. G. y Suárez Bedolla, B. I.

Diagnóstico de la construcción teórica de investigaciones en Comunicación en México

\section{DISCUSIÓN}

Aun cuando el objetivo general de la maestría en comunicación en la UNAM es "proporcionar al alumno una formación amplia y sólida en un campo de conocimiento, iniciarlo en la investigación, formarlo para la docencia y/o desarrollar en él una alta capacidad para el ejercicio profesional" y el objetivo general de los estudios del doctorado es "proporcionar al alumno una formación sólida para desarrollar investigación que produzca conocimiento original y ofrecer una rigurosa preparación para el ejercicio académico y/o profesional" llama la atención que los datos apunten a dos debilidades teórico-metodológicas importantes: la falta de una explicitación de las hipótesis en casi 4 de cada 10 tesis y la falta de crítica teórica en poco más del $70 \%$ de los trabajos.

La falta de hipótesis o premisas mínimas de la investigación, como se mencionaba anteriormente, impide una visualización del diseño del proyecto y la orientación que tomarán los resultados que se obtengan. Mientras que, la escasa crítica teórica podría suponer una inexistente autonomía intelectual que, en los niveles estudiados, se sugiere necesaria para la producción de conocimiento original y para la incursión sólida en el quehacer científico.

Otro aspecto interesante que ilumina los resultados es que las tesis de posgrado dedicadas a la comunicación abarcan una gran variedad de temas y líneas de investigación, lo cual nos habla sobre la vastedad y flexibilidad misma de la disciplina. Sin embargo, los objetos de estudio parecieran dejar de lado a los actores como punto central de análisis y se siguen concentrando mayoritariamente en los mensajes/expresiones. En el mismo sentido, también se observa que cobran relevancia las perspectivas centradas en el estudio de las representaciones y los instrumentos.

Cabe preguntarse por qué la falta de relevancia de los actores como punto central dentro de las tesis de posgrado, incluso al hablar de audiencias se estudian en su calidad de receptores y no desde su papel como actores sociales. Quizás siga privando la metáfora del impacto de los medios y de los mensajes. En cuanto al acento en el estudio de los mensajes, aparece cierta inclinación en el posgrado a formar especialistas en lenguaje.

Se observa una fuerte presencia de literatura norteamericana y europea, en comparación con autores y literatura latinoamericana. Este dato podría señalar cierta inconsistencia en la formulación teórica de los problemas de investigación, dado que, si los objetos de estudio se ubican fundamentalmente en el contexto nacional mexicano, la realidad nacional podría no ajustarse a reflexiones teóricas que obedecen a contextos externos. Esto de ninguna manera significa que las teorías tengan nacionalidad, pero sí es sintomático de una falta de consolidación de trabajo teórico en autores mexicanos y/o de diálogo teórico entre connacionales. 
Gutiérrez Morales, I. M.; Aguilar Ramírez, L. A.; González Chaparro, I. L.; Pérez

Lima, A. M.; Sandoval Pantoja, M. G. y Suárez Bedolla, B. I.

Diagnóstico de la construcción teórica de investigaciones en Comunicación en México

En cuanto al aspecto metodológico, los trabajos de comunicación en el Posgrado de Ciencias Políticas y Sociales presentan una preferencia por los abordajes cualitativos, en los cuales se distinguen técnicas clásicas tales como la entrevista, el análisis del discurso y la observación; mientras que los trabajos puramente cuantitativos parecen perder terreno. Siguen apareciendo como parte de las metodologías mixtas, que con un $30.3 \%$ empiezan a cobrar visibilidad.

El bajo porcentaje de investigaciones cuantitativas podría sugerir la preferencia de ciertos macos teóricos/conceptual o la visión de que la comunicación como objeto de estudio está ligado a procesos complejos de construcción de la realidad y una marcada influencia de la subjetividad humana, mismos procesos que demandan para su comprensión un enfoque cualitativo. Otra posible explicación podría ser el escaso empleo de técnicas cuantitativas por su grado de rigurosidad y de tratamiento matemático.

Sobre la recurrencia a determinados autores y obras se explica por la necesidad de justificar la investigación basándose en la autoridad intelectual de autores consolidados en el campo de las ciencias sociales (particularmente desde la disciplina sociológica), de la comunicación, y en específico, en el Posgrado de Ciencias Políticas y Sociales de la UNAM.

Haciendo un cruce de variables, se encontró que las correlaciones entre los reactivos, en todos los casos fue débil e imperfecta (de 0.3 a 0.1 y de -0.3 a -0.1). Esto puede explicarse estadísticamente a partir de la dispersión tan pronunciada de líneas, temas, autores y obras, siendo el eje de comunicación y cultura el que agrupan mayores líneas temáticas y un fuerte acento en la investigación cualitativa.

\section{CONCLUSIONES}

La comunicación como disciplina ha transitado, desde sus orígenes como el estudio de los medios de comunicación de masas (mass media research), hasta un saber interdisciplinario que, a partir de procesos comunicativos, es capaz explicar fenómenos sociales, económicos, políticos, educativos o culturales con absoluto rigor científico.

Hoy en día, la investigación en comunicación en México exhibe una amplitud importante de temas, objetos de estudio, metodologías y técnicas que, lejos de asumirse como un factor de dispersión o fragmentación del saber comunicativo, debe valorarse en su capacidad de integración disciplinaria para entender y atender problemáticas complejas, como las que en la actualidad emergen en el contexto nacional e internacional.

Esta investigación revela que un mapa teórico de los estudios en comunicación debe considerar un importante acento en la transición del análisis de contenidos mediáticos al estudio de discursos proferidos en todos los ámbitos sociales, y en la emergencia de asuntos de relevancia global como la economía política de la industria 
Gutiérrez Morales, I. M.; Aguilar Ramírez, L. A.; González Chaparro, I. L.; Pérez Lima, A. M.; Sandoval Pantoja, M. G. y Suárez Bedolla, B. I.

Diagnóstico de la construcción teórica de investigaciones en Comunicación en México

de la comunicación, los estudios de género, la comunicación política, la significación de los fenómenos culturales, los procesos comunicativos interpersonales y organizacionales y las innovaciones tecnológicas, desde la perspectiva sociocultural más que técnica.

La presente investigación también sugiere que, a pesar de la vastedad de insumos, la autonomía teórica de los investigadores de la comunicación en México es un área de oportunidad. Crear modelos o teoría comunicativas es una tarea que debe atenderse a partir del impulso y la consolidación del trabajo investigativo, el diálogo teórico entre pares y la conformación de comunidades científicas.

\section{REFERENCIAS}

American Psychological Association. (s.f.). APA Style. Recuperado de: http://www.apastyle.org/

Bunge, M. (1993). La ciencia, su método y su filosofía. Argentina: Ediciones Siglo Veinte.

Campos Arzeta, M. (2009). La comunicación en México. Una agenda de investigación. Archipiélago, 64, 30.

Einstein, A. (1981). Mis ideas y opiniones. Barcelona: Bosch.

Fuentes Navarro, R. (2014). Postgrados e Investigación en comunicación en México ¿Estancamiento o evolución de la desarticulación múltiple? Comunicación y sociedad, 13-51.

González Reyna, M. S. (2015). Historias y aportes sociales de la investigación de la comunicación en México. Los campos de estudio de la comunicación en el posgrado de ciencias políticas y sociales de la UNAM. Memorias XXVII AMIC (pp. 3142-3156). Querétaro: AMIC.

Karam Cárdenas, T. (2009). Elementos para el análisis del campo académico de la comunicación en México. En J. Galindo Cáceres, T. Karam Cárdenas, \& M. Rizo García, Comunicología en construcción (pp. 71-94). México, México: Universidad Autónoma de la Ciudad de México.

Karam Cárdenas, T. (2009). Notas sobre el estado de la investigación de la comunicación en México. En J. Galindo Cáceres, T. Karam Cárdenas, \& M. Rizo García, Comunicología en construcción (pp. 385-426). México, México: Universidad Autónoma de la Ciudad de México.

Lyons, J. (1997). Semántica lingüística: una introducción, Barcelona: Paidós.

Palmer, F. R. (1978). La semántica: una nueva introducción. México: Siglo XXI. 
Gutiérrez Morales, I. M.; Aguilar Ramírez, L. A.; González Chaparro, I. L.; Pérez Lima, A. M.; Sandoval Pantoja, M. G. y Suárez Bedolla, B. I.

Diagnóstico de la construcción teórica de investigaciones en Comunicación en México

Popper, K. (1994) Conocimiento objetivo. Madrid: Tecnos.

Schlanger, J. (1983). L'activité theorique, París: Librairie Philosophique.

Serrano, M. (1982). Teoría de la Comunicación. I. Epistemología y análisis de la referencia. Madrid: A. Corazón Editor.

Rodríguez, J. A. M. y Fuentes, M. B. F. (2012). “Revistas académicas de Comunicación en México: evaluación inicial de su contribución disciplinaria" en Elegir dónde publicar, pp. 25-34.

Ullmann, S. (1991). Semántica: introducción a la ciencia del significado. Madrid: Taurus.

\section{AUTORES:}

\section{Irma Mariana Gutiérrez Morales}

Doctora en Ciencias Políticas y Sociales por la Universidad Nacional Autónoma de México. Profesora de tiempo completo de las Licenciaturas en Comunicación y Pedagogía y de la Maestría en Comunicación de la Facultad de Estudios Superiores Acatlán, UNAM. Fue jefa del Departamento de Desarrollo e Investigación en Comunicación y Estudios Culturales en la Unidad de Investigación Multidisciplinaria de la misma Facultad. Es Candidata a Investigador Nacional por el Consejo Nacional de Ciencia y Tecnología de México. Ha publicado diversos artículos en revistas arbitradas e indexadas y dictado conferencias sobre sus proyectos de investigación, mismos que giran en torno a tres líneas: Comunicación Educativa, Estudios Culturales y Estudios del Discurso.

mariana_gmx@yahoo.com

Luis Antonio Aguilar Ramírez, Irma Leticia González Chaparro, Angélica Montserrat Pérez Lima, María Guadalupe Sandoval Pantoja y Benito Ilich Suárez Bedolla

Maestrantes en Comunicación por la Universidad Nacional Autónoma de México. Becarios del Consejo Nacional de Ciencia y Tecnología de México y participantes del proyecto de investigación "Diagnóstico de la construcción teórica de investigaciones en comunicación en México". Actualmente, cada uno desarrolla un proyecto de investigación propio en el campo de la comunicación, en las líneas de Comunicación en las organizaciones, Divulgación de la ciencia, Estudios de recepción, Innovaciones tecnológicas y sociedad y Comunicación, cultura y procesos educativos.

1_aguilaramirez@yahoo.com.mx

igtrabajo@yahoo.com.mx

ampl.monrock@gmail.com

sandovalmagu@gmail.com

benitoilich@gmail.com 